\title{
Icon Size Effect on Selection Speed and Accuracy
}

\author{
Kleddao Satcharoen
}

\begin{abstract}
The objective of this research is to evaluate the effect of icon size on selection speed and accuracy. An experiment was designed for investigation of icon selection on a mobile device. A set of 20 icons, with a combination of six icon sizes (16pix to 96pix square), was used to generate 120 trials per subject $(n=52)$. Selection time (seconds) and accuracy (accurate/inaccurate) were collected. Data was analyzed using chi-square tests. Analysis of TimexAccuracy showed that $2 \mathrm{~s}$ had the lowest inaccuracy rates $(.84 \%)$, while $5 \mathrm{~s}+$ had the highest inaccuracy rates $(\mathbf{8 . 8 6 \%})$. When considering Icon Size $\times$ Time $\rightarrow$ Accuracy showed that there were significant differences for most groups. The highest inaccuracy rates were seen for small icons (48pix and smaller), and inaccuracy climbed at higher Time. The implication of this study is that icon size can negatively influence selection speed and that higher selection speed was associated with lower accuracy as well.
\end{abstract}

Index Terms-Icon size, icon selection, accuracy, response time.

\section{INTRODUCTION}

Although icons are not the most frequently investigated user interface tool, their importance should not be overlooked. Icons allow users to rapidly and efficiently execute commands on their devices. They also offer mnemonic associations that assist in remembering how to execute these commands [1]. The use of graphical representations for specific tasks that users want to complete on their computing devices, such as launching programs or even interacting with other users, is now ubiquitous, especially on app-based small-screen computing devices [2]. Icon representation can even be used to solve problems like how to localize languages and settings when there is no shared language between the user and the system default [3] or overcoming challenges to communications in emergencies [4]. However, icon-based systems are not perfect for all users. For example, older users and those with visual impairments may struggle to use icon-based interaction systems, especially those that make use of small icons or cramped screen space [5]. Similarly, those that are less familiar with the representation system, and who therefore have a higher semantic distance between what is seen and what is intended, may take longer to identify correct icons [6]. Thus, it is essential to consider the trade-offs that icon-based systems entail and how the icon size influences their usability. The objective of this research was to evaluate the effect of icon size on selection speed and accuracy using an experimental approach.

Manuscript received May 9, 2020; revised August 2, 2020.

Kleddao Satcharoen is with Department of Computer Engineering, Faculty of Engineering. King Mongkut's Institute of Technology Ladkrabang, Bangkok, Thailand (e-mail: kleddao.sa@ kmitl.ac.th).

\section{LITERATURE REVIEW}

\section{A. Human Computer Interaction}

Human-computer interaction (HCI) can be described generally as the processes by which a computing system and the human user exchange information, using the computing system's input and output systems, processor and memory and the human's sensory-motor and cognitive systems [7]. The purpose of HCI systems is primarily to enable the human user to achieve specific ends and goals through manipulation of the computer's systems and processors. Although there are many different interaction systems that can be used in modern computing (for example, those that use haptic feedback or rely on eye-scanning or gestures), this research was mainly concerned with the graphic user interface (GUI), which remains one of the most commonly used interface designs [8]. GUIs are typically designed using visual and touch or pointing input (such as a mouse) to allow a user to execute commands and perform other tasks, and are used in both mobile and traditional computing systems.

\section{B. Computer Icon}

The computer icon (or simply icon) is a graphical object that allows the user to execute some command within a computer system, for example accessing a file or launching a program or app [8]. Although icons do not have an inherent requirement for semantic meaning, they typically are designed for uniqueness and semantic depth to enable users to create associations between the image and what it represents [1], [6], [9]. Icons were introduced in the 1980s as a part of the early GUIs used in personal computers, but they were used side-by-side with command lines (what one author has called 'direct manipulation') [10]. Initial research, which took place in the context of relatively skilled users engaged in workplace rather than recreational or personal computer usage, did not suggest that there was much advantage to the use of icons compared to command-line or menu-based interfaces [10]. However, that changed dramatically with the implementation of small-screen computing, where miniaturized interfaces like mobile phones and tablet devices left much less room for interface design [11], [12]. Under these conditions, icon-based representation became more effective than alternatives such as command-line interfaces, although there is not yet a complete understanding of how the miniaturization of interfaces and small screens influences usability [11]. Thus, there is still room for investigation of the role of icon size and other characteristics on the usability of icons.

\section{Icon Size and Selection Speed and Accuracy}

This research addresses the interaction of icon size, selection speed, and accuracy. Icon size refers to the display 
size of the icon on the screen (typically measured in pixels) [8]. Accuracy refers to the frequency with which the user successfully matches the icon with the prompt (thereby executing the desired command) [9]. Selection speed, also commonly called response time, refers to the amount of time required for the user to make their selection [12]. Several previous studies have evaluated the interaction of icon size, selection speed, and selection accuracy. While some of these studies have only focused on icon size as a variable, others have investigated different sets of characteristics.

\section{1) Icon size and selection speed}

One of the earliest studies that investigated the effect of icon size on selection speed (and accuracy as well) was that of Schröder and Ziefle [12], who conducted an experiment similar to the one used in this research. In this experiment $(n$ $=20$ ), users were asked to select specific icons from 20 presented to them on a mobile phone screen, and icon selection speed and accuracy were assessed. Various parameters of the icons, including the set size and the icon display size, were manipulated. Analysis revealed that selection speed (coded as reaction time) was fastest for icon size: display size ratios of 1:50, regardless of the actual size. However, the actual size of the icon and screen had an inverse effect on selection speed; larger icons and displays were associated with faster reaction times.

A second study investigated icon size and grid size on the speed of menu selection on a smartphone [13]. These authors used $3 \times 2$ experiment design (grid size $(3 \times 4,3 \times 3$, and $2 \times 3$ ) and icon size $(48 \times 48$ pixels and $60 \times 60$ pixels $)$, with a sample of 30 students. They tested only the time required to select the icon. Their finding showed that the icon size itself did not have a significant effect on selection time, although grid size did have a significant effect. There was also no interaction effect. Therefore, the study did not show that icon size had an effect on selection speed.

Another study investigated grid layout and icon size in the speed and accuracy of icon selection in a mobile interface in an experimental sample of mobile phone users $(n=40)$ [14]. The authors used a $4 \times 3$ experimental design to manipulate layout and icon ratio on a fixed-size screen, resulting in icon sizes ranging from $4.77 \mathrm{~mm}$ to $17.33 \mathrm{~mm}$ for a total of 96 trials. Authors investigated touch performance (selection accuracy or hit rate), selection time, and selective perception of the icon size suitability. There was no time limitation, but authors did record the amount of time required to select the icon. They found that an increase in icon ratio resulted in an increased amount of time required to select the icon, probably because users had to move through more icon pages to identify the correct icon.

An earlier study in the present research project showed that size did have an effect on selection speed [15]. This study used an experimental design in which icon sizes were manipulated across a total of 60 trials. The sample $(n=50)$ was drawn from a population of British students. The experimental apparatus used a laptop computer. Subjects were used to match a single displayed icon to a text-based prompt, which was presented in a set of 10 possible prompts. The outcomes showed that in general, smaller icon sizes were associated with slower selection speeds. However, there was a break at $48 \mathrm{x} 48$ pix, and icons at and above this size had similar selection times. Thus, this study showed that there was a significant negative effect of icon size on selection speed, but only at lower than 48pix.

Taken together, these studies suggest that smaller icons will take longer to select, although this finding depends on the experimental design. Therefore:

Hypothesis 1: Larger icon sizes will have faster selection speeds.

Hypothesis 2: Icon selection speed will influence accuracy.

\section{2) Icon size and accuracy}

Schröder and Ziefle [12] also evaluated the effect of icon size on selection accuracy. They found that most icon sizes did not have a significant effect, but that the smallest icon display size had a significant negative effect on the selection accuracy. However, icons larger than $16 \times 20$ pix were deemed to be large enough for efficient search, since the effect of larger icons eroded rapidly [12].

Another study, which investigated icon ratios rather than fixed icon sizes, also found that larger icon sizes were associated with faster selection [14]. Specifically, they showed that the icon design resulting in a 0.9 icon ratio (the largest icons regardless of layout) had a significant positive effect on hit rate or accuracy compared to the smaller icon ratios. Thus, this was the opposite of the effect observed in this apparatus for icon selection speed. This inverse effect is most likely related to the design of the apparatus, as it did not result in all icons displaying in a single screen.

Earlier studies in this research project also demonstrated an effect on selection accuracy rates [15]. In this study, it was demonstrated that the percentage of inaccurate answers fell as the icon size rose; while $16 \times 16$ pixels icons had an approximately $7 \%$ rate of inaccuracy, $64 \times 64$ pixels icons and larger were associated with $1 \%$ inaccuracy rates or lower.

These studies strongly suggest that larger icons are associated with more accurate selection. Therefore:

Hypothesis 3: Larger icon sizes will have higher selection accuracy rates.

\section{3) Selection speed and accuracy}

One study investigated icon characteristics (icon entropy and concreteness) and time constraints on selection accuracy [16]. An experiment involving 400 Thai engineering undergraduates was conducted. This experiment used varying icon characteristics and three-time constraints $(5 \mathrm{~s}$, $10 \mathrm{~s}$ and $15 \mathrm{~s})$. Under the longer time constraints (10s/15s) there was no difference in selection accuracy, but under the 5 s time constraint the effect of high entropy on accuracy was significant. A second study investigated background and icon entropy under timed and untimed conditions (3s/no time limit) in an experiment involving 100 Thai undergraduates [17]. This study found that icon entropy did not have an effect on accuracy in untimed trials, but that there was a significant negative effect on accuracy in the timed trials. The implication of these studies is that time constraints could impose cognitive processing challenges that could exacerbate the negative effects of other icon characteristics (for example small size), as has been shown for the interaction of icon entropy and time. Thus, while these studies are limited, they suggest that:

Hypothesis 4: Icon size will have an effect on the interaction of selection speed and accuracy. 


\section{Methodology}

An experiment was conducted in a sample of 52 participants $(n=52)$, who were recruited from a university setting. These participants, who ranged in age from 20 to 44 years, were studying for Bachelor or Master's degrees. The experimental apparatus used a mobile app designed for the experiment, which automatically logged attempts and collected data. The experimental task was a matching task. Participants were asked to identify 20 different easily recognizable icons from sound prompts. Participants repeated the matching task six times with icons at different sizes, with a total of 120 trials per participant. (Icon sizes and imagery are summarized in Table I.) Respondents were timed but no time limitation was placed on completion of the task. Respondent time and accuracy was recorded for each trial. Analysis was conducted using Pearson chi-square tests.

\section{FINDINGS AND DISCUSSION}

\section{A. Findings}

The first test addressed selection speed and accuracy (Table II). Results showed that there were similar levels of accuracy for $1 \mathrm{~s}, 3 \mathrm{~s}$ and $4 \mathrm{~s}$ conditions. The cross-tabulation showed that overall accuracy ratios for these categories (representing inaccurate to accurate results) were also similar, ranging from $1.03 \%$ and $1.62 \%$. Results in the $2 \mathrm{~s}$ category were the most accurate $(.83 \%)$, while results in the $5 \mathrm{~s}+$ category were highest $(8.7 \%)$. This indicates that a slower selection speed was associated with a lower accuracy rate.

TABLE I: SUMMARY OF THE TEST CONDITIONS

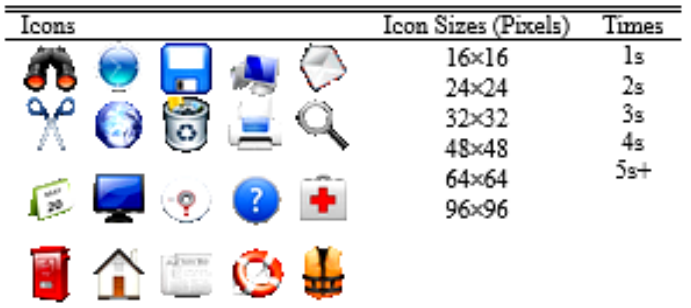

TABLE II: SELECTION SPEED - ACCURACY

\begin{tabular}{cccc}
\hline \hline Time (Seconds) & Chi-Square & $\mathrm{p}$ & $\begin{array}{c}\text { Accuracy ratio } \\
\text { (Inaccurate/Accurate) (\%) }\end{array}$ \\
\hline $1 \mathrm{~s}$ & 6.477 & .262 & 1.03 \\
$2 \mathrm{~s}$ & 16.748 & $.005^{* 8}$ & .83 \\
$3 \mathrm{~s}$ & 2.323 & .803 & 1.41 \\
$4 \mathrm{~s}$ & 9.978 & .076 & 1.62 \\
$5 \mathrm{~s}+$ & 12.481 & $.029^{*}$ & 8.7 \\
\hline \hline
\end{tabular}

Note: Dependent Variable: Accuracy; ${ }^{*} \mathrm{p}<.05 * * \mathrm{p}<.01 * * * \mathrm{p}<.001$

The second test investigated the joint effect of icon size and selection speed on accuracy (Table III). This analysis shows that there are some significant effects of icon size on accuracy. In general, accuracy is higher for the larger icons (above $48 \times 48$ pixels). However, it can also be seen that the accuracy is highest in lower time periods (1s and $2 \mathrm{~s}$ ) and lowest in longer time periods (especially $5 \mathrm{~s}+$ ). The results also show that larger icon sizes were associated with faster selection time; for example, while only $20.1 \%$ of $16 \times 16$ pixels icons were selected in $1 \mathrm{~s}$ or $2 \mathrm{~s}, 33.4 \%$ of $64 \times 64$ pixels and $41 \%$ of $96 \times 96$ pixels icons were selected in the same time period. Total accuracy ratios for icon size confirm that on average, icon sizes of $48 \times 48$ pix and below are less accurate than average $\chi 2$.

TABLE III: SELECTION SPEED - ACCURACY

\begin{tabular}{|c|c|c|c|c|c|c|c|c|}
\hline \multirow[t]{3}{*}{ Size } & \multirow[t]{3}{*}{$\chi^{2}$} & \multirow[t]{3}{*}{$\bar{P}$} & \multicolumn{5}{|c|}{ Accuracy Ratio (\%) } & \multirow{3}{*}{$\begin{array}{l}\mathrm{AR} \\
(\%)\end{array}$} \\
\hline & & & \multicolumn{5}{|c|}{$\%$ of Trails Selected } & \\
\hline & & & $1 \mathrm{~s}$ & $2 \mathrm{~s}$ & $3 \mathrm{~s}$ & $4 \mathrm{~s}$ & $5 \mathrm{~s}$ & \\
\hline \multirow[t]{2}{*}{16} & 35.651 & $.000^{* * * *}$ & 0 & 3.3 & 2.2 & 3.2 & 11.7 & 5.2 \\
\hline & & & 2.5 & 17.6 & 31 & 21.1 & 27.9 & \\
\hline \multirow[t]{2}{*}{24} & 52.519 & $.000^{* * * *}$ & 0 & 1.3 & 1.5 & 1 & 11.5 & 2.9 \\
\hline & & & 3.8 & 23.1 & 37.9 & 19.3 & 15.9 & \\
\hline 32 & 23.707 & $.000^{* * *}$ & $\begin{array}{l}0 \\
5\end{array}$ & $\begin{array}{l}0.36 \\
268\end{array}$ & $\begin{array}{l}1.3 \\
38.2\end{array}$ & $\begin{array}{c}0.6 \\
17.2\end{array}$ & $\begin{array}{c}6 \\
12.8\end{array}$ & 1.4 \\
\hline \multirow[t]{2}{*}{48} & 17.760 & $.001^{* * *}$ & 4.1 & 0.63 & 1.5 & 3.5 & 6.6 & 2.3 \\
\hline & & & 4.7 & 30.7 & 37.6 & 13.9 & 13.1 & \\
\hline \multirow[t]{2}{*}{64} & 3.78 & 0.44 & 0 & 0.35 & 1.2 & 0.6 & 2.0 & 0.9 \\
\hline & & & 5.7 & 27.7 & 39.7 & 17.5 & 9.4 & \\
\hline \multirow[t]{4}{*}{96} & 27.696 & $.000^{* * * *}$ & 1.5 & 0.3 & 0.9 & 0.8 & 7.8 & 1.2 \\
\hline & & & 6.3 & 34.7 & 41.3 & 11.6 & 6.2 & \\
\hline & \multirow{2}{*}{\multicolumn{2}{|c|}{ All icon sizes }} & 1.0 & 0.8 & 1.4 & 1.6 & 8.7 & 2.3 \\
\hline & & & 28 & 26.8 & 37.6 & 16.8 & 14.2 & \\
\hline
\end{tabular}

Note: Dependent Variable: Accuracy; ${ }^{*} \mathrm{p}<.05 * * \mathrm{p}<.01 * * * \mathrm{p}<.001$

The overall finding can be stated as follows. First, there is a significant effect of icon speed on accuracy, but the direction is unexpected: More rapid selection was associated with higher accuracy. Second, larger icons were associated with faster selection speed and accuracy. Finally, there is an interaction between icon size and selection time that influenced accuracy. Thus, $\mathrm{H} 1$ and $\mathrm{H} 4$ are rejected due to the direction, but $\mathrm{H} 2$ and $\mathrm{H} 3$ are accepted.

\section{B. Discussion}

These findings are in the main as expected from the literature, given that they do indicate that a) icon size influences both selection speed and selection accuracy [1], [3], [14]. However, there are some variances that were not expected. The main variance is that there was a high rate of inaccuracy in the $5 \mathrm{~s}+$ time period. This is similar to what has been observed in one other study, where a higher icon ratio (resulting in a larger subjective icon size) also increased the icon selection time [3]. This may be due to the use of a mobile interface, where a larger icon size could necessitate paging through or scrolling around the interface. Part of the novelty of this research lies in its demonstration of relatively high inaccuracy rates for very high search times. This suggests that high search times could be associated with inadequate cues or too high a level of semantic distance for users to overcome [2], [10]. Under these conditions, it may be useful to use movement cues (for example, twist/pulse indicators) to identify icons for users [4].

\section{CONCLUSION}

The objective of this research was to evaluate the effect of icon size on selection speed and accuracy. The experimental findings showed that icon size does have the expected effects on selection speed (reducing it) and accuracy (increasing it). However, the interactions between icon selection speed and accuracy were not as expected. In fact, while it was expected from prior studies that selection accuracy rates would be higher at slower speeds, in practice, the slowest selections $(5 \mathrm{~s}+)$ were also the least accurate. It likely that this finding occurred because users who could easily identify an icon would do so quickly, leaving users that could not identify the icon readily with a longer search pattern and increased inaccuracy risk. However, the study did achieve the objective 
of investigating icon size.

The implication of this finding is that if users do struggle with slow icon selection or selection inaccuracy, it may be helpful to increase the icon display size. However, it should be noted that accuracy rates (high) and overall selection speed ( $1 \mathrm{~s}$ or $2 \mathrm{~s}$ for most trials) were already good. Therefore, it may not be worth changing the icon size proactively. Instead, it may be more useful as a per-user recommendation or for applications designed for users that are thought to have particular issues in this area, such as the elderly or vision-impaired.

This study was not designed to identify why users may struggle with icon identification, although other studies have suggested problems including high semantic distance between the icon designer and user and visual and cognitive impairments that could prevent or limit ability to recognize icons. Thus, there are still opportunities for research into optimizing icon design to overcome these challenges, especially in mobile and small-screen computing contexts.

\section{CONFLICT OF INTEREST}

The authors declare no conflict of interest.

\section{AUTHOR CONTRIBUTIONS}

As this research have only one author, the contribution of this research could clarify by first designing the experimental what the processes should be included. Then generated the questionnaire for participants and collecting the data. After received all the data from participants who participated, then analyzed data, and wrote the paper with editing and prepared for the final version.

\section{ACKNOWLEDGMENT}

The author would like to Thanks all the participants who participated this research. I appreciate your time you're your honesty and your kind, without you this research would not be successful.

\section{REFERENCES}

[1] S. Isherwood, "Graphics and semantics: The relationship between what is seen and what is meant in icon design," in Proc. International Conference on Engineering Psychology and Cognitive Ergonomics, 2009, pp. 32-42.

[2] J. Cuello and J. Vittone, Designing Mobile Apps, 2013.

[3] A. M. Awwad and W. Slany, "Automated bidirectional languages localization testing for android apps with rich GUI," Mobile Information Systems, 2016.

[4] A. Patton et al., "Using icons to overcome communication barriers during emergencies: A case study of the Show Me interactive tools," Visible Language, vol. 49, no. 1/2, pp. 80-95, 2015.
[5] J. Zhou et al., "Older adults use of smart phones: An investigation of the factors influencing the acceptance of new functions," Behaviour and Information Technology, vol. 33, no. 6, pp. 552-560, 2014.

[6] S. McDougall and S. Isherwood, "What's in a name? The role of graphics, functions, and their interrelationships in icon identification," Behavior Research Methods, vol. 41, no. 2, pp. 325-336, 2009.

[7] A. Dix, "Human-computer interaction," in Encyclopedia of Database Systems, L. Liu and M. T. Özsu, Eds. Springer, 2009.

[8] B. Shneiderman et al., Designing the User Interface: Strategies for Effective Human-Computer Interaction, Pearson, 2016.

[9] S. J. Isherwood et al., "Icon identification in context: The changing role of icon characteristics with user experience," Human Factors, vol. 49, no. 3, pp. 465-476, 2007.

[10] I. Benbasat and P. Todd, "An experimental investigation of interface design alternatives: Icon vs. text and direct manipulation vs. Menus," International Journal of Man-Machine Studies, 1993.

[11] L. Punchoojit and N. Hongwarittorrn, "Usability studies on mobile user interface design patterns: A systematic literature review," Advances in Human-Computer Interaction., 2017.

[12] S. Schröder and M. Ziefle, "Icon design on small screens: Effects of miniaturization on speed and accuracy in visual search," in Proc. the Human Factors and Ergonomics Society, pp. 656-660, 2006.

[13] V. Tadayin et al., "The effect of icon size and grid size on smartphone menu selection," ACM International Conference Proceeding Series, pp. 64-68, 2018

[14] Y. Im et al., "Investigation of icon design and touchable area for effective smart phone controls," Human Factors and Ergonomics in Manufacturing and Service Industries, vol. 25, no. 2, pp. 251-267, 2015.

[15] K. Satcharoen, "Does size matter in the speed and accuracy on image identification?" in Proc. International Conference on Control, Automation and Systems, 2017, pp. 878-882.

[16] K. Satcharoen, "The effect of icon entropy, icon concreteness and time on human selection accuracy," in Proc. 2019 IEEE 4th International Conference on Computer and Communication Systems (ICCCS), 2019.

[17] K. Satcharoen, "Effect of entropy in icons and background on selection accuracy," in Proc. 2018 3rd International Conference on Computer and Communication Systems (ICCCS), 2018.

Copyright (C) 2020 by the authors. This is an open access article distributed under the Creative Commons Attribution License which permits unrestricted use, distribution, and reproduction in any medium, provided the original work is properly cited (CC BY 4.0).

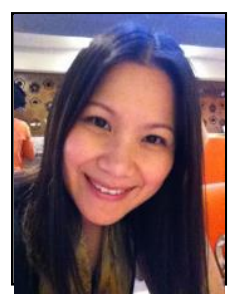

Kleddao Satcharoen was born in May 1978, Thailand. She earned her DPhil in computing (2017) and MSc in computing (2003) from the University of Buckingham, UK, and a MA in political science (2005) from Ramkhamhaeng University, Bangkok, Thailand. She also holds the BSc in management technology (2000) from King Mongkut's Institute of Technology Ladkrabang (KMITL), Bangkok, Thailand.

Since 2004, she has been working as a lecturer and now she is an assistant professor in Faculty of Engineer, Department of Computer Engineering at King Mongkut's Institute of Technology Ladkrabang, Bangkok, Thailand. She currently teaches 4 main courses: human computer interaction, user interface and innovative design concept, system analysis and design, and web technology. Therefore, her interest is on human computer interaction area, specific in icon characteristics, perception and algorithmic/programmatic techniques for icon characteristics measurement. 